\title{
A functional peptidoglycan hydrolase characterized from T4SS in 89K pathogenicity island of epidemic Streptococcus suis serotype 2
}

\author{
Qiu Zhong ${ }^{1,2}$, Yan Zhao ${ }^{1}$, Tian Chen ${ }^{1}$, Supeng Yin ${ }^{1}$, Xinyue Yao ${ }^{1}$, Jing Wang ${ }^{1}$, Shuguang Lu', Yinling Tan ${ }^{1}$,
} Jiaqi Tang ${ }^{3}$, Beiwen Zheng ${ }^{4}$, Fuquan $\mathrm{Hu}^{1^{*}}$ and Ming $\mathrm{Li}^{1^{*}}$

\begin{abstract}
Background: Streptococcus suis serotype 2 (S. suis 2) has evolved efficient mechanisms to cause streptococcal toxic shock syndrome (STSS), which is a new emerging infectious disease linked to S. suis. We have previously reported that a type IV secretion system (T4SS) harbored by the specific 89K pathogenicity island (PAI) of S. suis 2 contributes to the development of STSS and mediates horizontal transfer of 89K. However, the 89K T4SS machinery assembly in vivo and in vitro is poorly understood, and the component acting directly to digest the bacterial cell wall needs to be identified.
\end{abstract}

Results: The virB1-89K gene product encoded in the $89 \mathrm{~K} \mathrm{PAl}$ is the only one that shows similarity to the Agrobacterium VirB1 component and contains a conserved CHAP domain that may function in peptidoglycan hydrolysis, which makes it a plausible candidate acting as a hydrolase against the peptidoglycan cell wall to allow the assembly of the T4SS apparatus. In the current study, the CHAP domain of VirB1-89K from S. suis 89K PAI was cloned and over-expressed in Escherichia coli, and its peptidoglycan-degrading activity in vitro was determined. The results indicated that the VirB1-89K CHAP domain can degrade the peptidoglycan layer of bacteria. Deletion of virB1-89K reduces significantly, but does not abolish, the virulence of $S$. suis in a mouse model.

Conclusions: The experimental results presented here suggested that VirB1-89K facilitates the assembly of 89K T4SS apparatus by catalyzing the degradation of the peptidoglycan cell wall, thus contributing to the pathogenesis of S. suis 2 infection.

Keywords: Streptococcus suis serotype 2, Streptococcal toxic shock syndrome, Type IV secretion system, Pathogenicity island, Assembly, Peptidoglycan hydrolase

\section{Background}

Streptococcus suis serotype 2 (S. suis 2), an important zoonotic pathogen worldwide, has evolved to be a serious problem over the past two decades [1-3]. It was reported that $S$. suis 2 only causes sporadic cases of human infection with a mortality of less than $10 \%[4,5]$. However, it emerged as the leading cause of two large-scale outbreaks of severe epidemics in China in 1998 and 2005, respectively [6]. The unusual outbreaks affected over 200 individuals and killed 52 of them $[7,8]$. Besides its large size and the associated

\footnotetext{
*Correspondence: hufuquan2009@aliyun.com; sotx7080@163.com 'Department of Microbiology, Third Military Medical University, Chongqing 400038, China

Full list of author information is available at the end of the article
}

high mortality rate, these two outbreaks are unique in that a large proportion of patients were victim to streptococcal toxic shock syndrome (STSS) [7]. Before that, STSS has been limited to disease caused by the group A streptococcus [9], S. suis (nongroup A) has not previously been linked to STSS.

To get insight into the high virulence of the S. suis isolates emerged in China, we previously decoded the whole genomic sequence of two epidemic strains (98HAH12 and 05ZYH33) isolated from the 1998 and 2005 Chinese outbreaks respectively, and identified a pathogenicity island (PAI) designated $89 \mathrm{~K}$ that is specific for Chinese outbreak isolates [10,11]. Subsequently, we provided genetic evidence showing that an $89 \mathrm{~K}$-borne type 
IV secretion system (T4SS) forms an important pathway for horizontal transfer of $89 \mathrm{~K}$ and secretion of some unknown pathogenic effectors that are responsible for STSS caused by the highly virulent $S$. suis 2 strains $[12,13]$. However, the $89 \mathrm{~K}$ T4SS assembly process in vivo and in vitro remains largely unknown.

There has long been a general lack of knowledge of T4SS functions and cellular localization in gram-positive bacteria [14]. It has been suggested that the assembly processes must be similar to or even simpler than those in gram-negative bacteria $[15,16]$. In the well-characterized model for the Agrobacterium tumefaciens VirB/D T4SS, the VirB1 component functions as a lytic transglycosylase that can digest the peptidoglycan layer of cell wall, thus facilitating the assembly of envelope-spanning protein complex of T4SS under temporal and spatial control $[17,18]$. Among the single operon composed of 15 genes that encodes the functional T4SS in S. suis $89 \mathrm{~K}$ PAI, only the virB1-89K gene product shows similarity to the Agrobacterium VirB1 component and contains a conserved cysteine, histidine-dependent amidohydrolases/ peptidases (CHAP) domain that may function in peptidoglycan hydrolysis [19]. We once proposed that VirB1-89K should function to punch holes in the peptidoglycan cell wall to allow the assembly of the T4SS apparatus [12]. However, we did not provide direct evidence to support this hypothesis.

In the present study, therefore, we expressed and purified the CHAP domain of VirB1-89K in Escherichia coli, and tested its putative peptidoglycan hydrolysis activity in vitro. Furthermore, an isogenic knockout mutant of virB1-89K and its complementary strain were used in a mouse infection model to assess the contribution of VirB1-89K to the virulence of $S$. suis outbreak strain. The experimental results indicated that VirB1-89K facilitates the assembly of $89 \mathrm{~K}$ T4SS apparatus by catalyzing the degradation of the peptidoglycan cell wall, thus contributing to the pathogenesis of T4SS in the S. suis.

\section{Results}

\section{Characterization of the CHAP domain of VirB1-89K}

On the negative strand of the 89K PAI in the genome of S. suis 05ZYH33 (GenBank accession number NC_009442), peptide encoded by $05 S S U 0968$ shows $18 \%$ similarity to the VirB1 component of the Agrobacterium T4SS and was designated VirB1-89K. Based on sequence analysis, VirB189K was predicted to contain a C-terminal CHAP domain (located between the amino acids 796 and 926) and an $\mathrm{N}$-terminal transmembrane domain, but lacks a signal sequence. The CHAP domain is broadly found in proteins from bacteria, phages, archaea, and eukaryotes of the Trypanosomidae family $[19,20]$. It has been proposed that the CHAP domain may function mainly in peptidoglycan hydrolysis [19]. The phylogenetic analysis of
VirB1-89K and its homologous proteins showed that VirB1-89K and N-acetylmuramoyl-L-alanine amidase probably originate from the same ancestor (Figure 1A).

Tertiary structure prediction showed that the CHAP domain of VirB1-89K belongs to the $\alpha+\beta$ structural class, with the $\mathrm{N}$-terminal half containing 3 predicted $\alpha$-helices and the $\mathrm{C}$-terminal half composed of 6 predicted $\beta$-strands (Figure 1B). Protein tertiary structure modeling revealed that this CHAP domain contains an putative active center composed of a conserved cysteine and a histidine (Figure 1C), these two invariant residues form the main part of the active site of CHAP domain containing proteins $[19,21,22]$. These results together with the above phylogeny analysis suggested that VirB1-89K may be an $\mathrm{N}$-acetylmuramyl-L-alanine amidase.

Expression and purification of the CHAP domain of VirB1-89K To figure out the function of VirB1-89K during the assembly of $89 \mathrm{~K}$ T4SS apparatus, a 411 bp DNA fragment containing the CHAP domain of VirB1-89K was cloned and over-expressed in E. coli as a C-terminally $\mathrm{His}_{6}$-tagged protein. The protein of interest was designated VirB189KCHAP. We found VirB1-89KCHAP was efficiently expressed after induction at $16^{\circ} \mathrm{C}$ (Figure 2A). The molecular mass of the expressed recombinant protein agreed well with a predicted size of $15.4 \mathrm{kDa}$. Although a majority of the VirB1-89KCHAP protein was present in the inclusion body fractions of crude cell lysates, sufficient soluble material was produced to recover useful amounts of active protein. Highly purified protein ( $>95 \%$ homogeneity) was prepared by $\mathrm{Ni}^{+}$affinity chromatography and gel filtration (Figure 2B). N-terminal sequencing results confirmed that the produced protein was indeed the CHAP domain of VirB1-89K.

\section{Lytic activity and biochemical characterization of VirB1-89KCHAP}

To determine the muramidase activity of the purified VirB1-89KCHAP protein, peptidoglycan hydrolase activity was analyzed by using zymography with $S$. suis peptidoglycan as substrate. After SDS-PAGE, the positive control hen egg white lysozyme, the negative control BSA protein, and the VirB1-89KCHAP protein could be seen after staining with Coomassie blue (Figure 3A). The gel was then stained with methylene blue to detect peptidoglycan hydrolase activity as a clear zone against a dark blue background. We noticed that VirB1-89KCHAP exhibited apparent enzyme activity as the positive control did, while the negative control BSA did not (Figure 3B). These zymography data suggested that the VirB1-89KCHAP protein could solubilize the cell wall of $S$. suis 2 .

In another set of experiments, the bacteriostatic activity of VirB1-89KCHAP was determined with slip diffusion method to confirm its peptidoglycan hydrolase activity. 


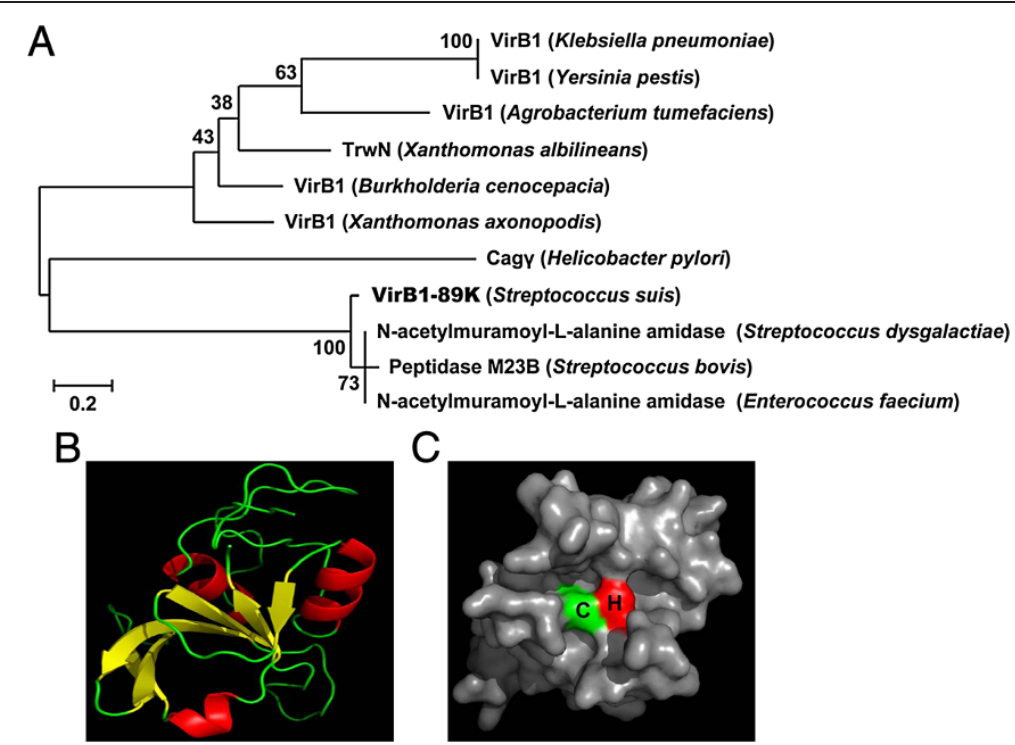

Figure 1 Sequence analysis of VirB1-89K. (A) Phylogenetic analysis of VirB1-89K. Sequence alignment and phylogenetic analysis of VirB1-89K homologs were performed using MEGA 5.1 software. Values at nodes indicate bootstrap values for 500 replicates. (B) Analysis of the tertiary structure of the CHAP domain of VirB1-89K by using the online server SWISS-MODEL. (C) Visualization of the surface active site of the CHAP domain by using PyMOLviewer, showing the cysteine residue in green and histidine in red.

We found that both the VirB1-89KCHAP protein and the hen egg white lysozyme could suppress the growth of S. suis 2, while the BSA control could not (Figure 3C).

To reveal the basic biological characteristics of VirB189KCHAP, we examined the optimum reaction condition of VirB1-89KCHAP by using Micrococcus lysodeikticus cells as substrate. Results showed that on increasing the $\mathrm{pH}$, peptidoglycan hydrolase activity of VirB1-89KCHAP increases and reaches maximum at pH 8.0 (Figure 4A). When the $\mathrm{pH}$ exceeds 9.0, the relative activity decreased sharply. VirB1-89KCHAP functions best at an optimal temperature of $40^{\circ} \mathrm{C}$. The enzyme activity rapidly declined at temperatures above $50^{\circ} \mathrm{C}$ and only $25 \%$ of the maximal activity was measured at $60^{\circ} \mathrm{C}$ (Figure 4B). From the thermal stability data, the relative activity is higher at $30^{\circ} \mathrm{C}$ than at $40^{\circ} \mathrm{C}$, suggesting that pre-incubation of VirB1-89KCHAP at $30^{\circ} \mathrm{C}$ causes lower decay in relative activity compared to the enzyme pre-incubated at $40^{\circ} \mathrm{C}$ (Figure $4 \mathrm{C}$ ). With increasing temperature, pre-incubation of VirB1-89KCHAP caused increasing decay in the relative activity of the enzyme.

\section{Role of VirB1-89K in bacterial virulence}

To assess the role of VirB1-89K in bacterial virulence, an isogenic knockout mutant of virB1-89K ( $\Delta$ virB1-89K)

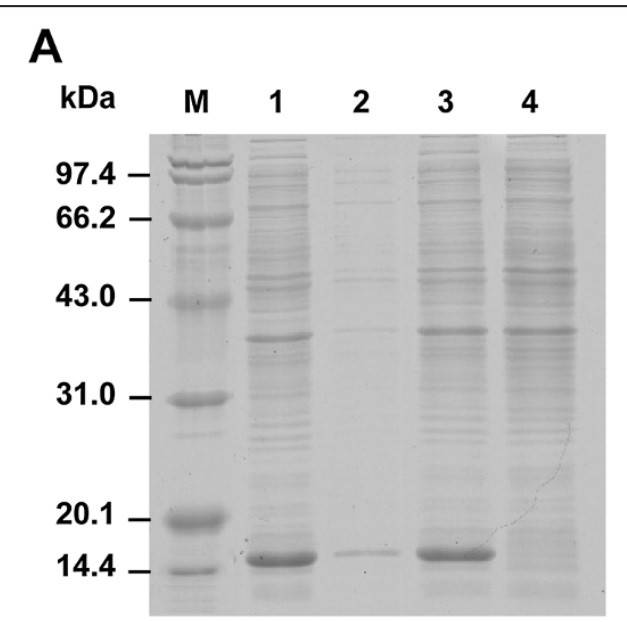

\section{B}

M VirB1-89KCHAP

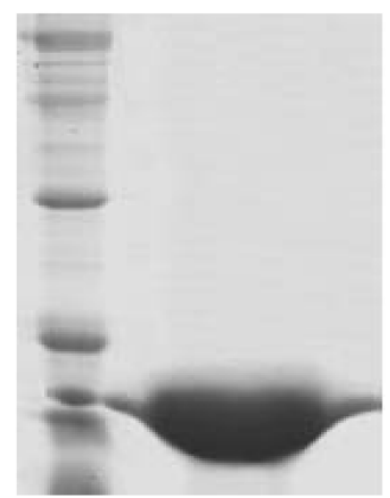

Figure 2 Over-expression and purification of VirB1-89KCHAP. (A) SDS-PAGE analysis (12\%) of the interest VirB1-89KCHAP protein expressed in E. coli. Lanes: 1, total crude extracts after IPTG induction; 2, sonicated supernatant of induced cells; 3, sonicated sediment of induced cells; 4 , noninduced cells. Protein size marker is indicated on the left. (B) The purified VirB1-89KCHAP protein after $\mathrm{Ni}^{+}$affinity chromatography and gel filtration. 


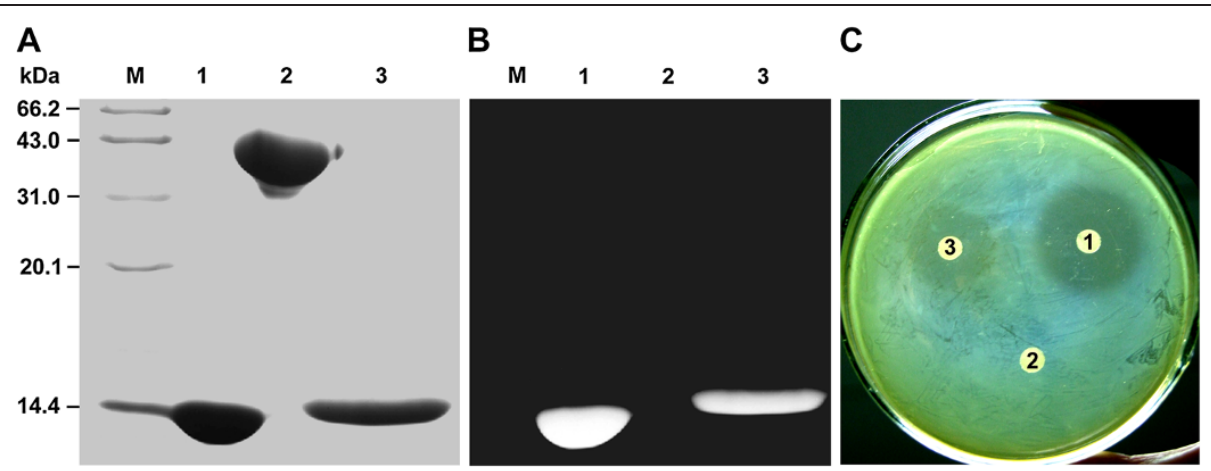

Figure 3 Lytic activity detection of VirB1-89KCHAP. Zymography analysis of peptidoglycan hydrolase activity of VirB1-89KCHAP. The gel was stained with Coomassie blue (A) and then overstained with Methylene blue (B). (C) Bacteriostatic activity of VirB1-89KCHAP. Proteins used: 1, hen egg white lysozyme; 2, BSA; 3, VirB1-89KCHAP.

constructed in our previous work and its complementary strain $C \Delta v i r B 1-89 K$ were subjected to experimental infection of mice [12]. We found that group of mice infected with the wild-type strain 05ZYH33 developed obvious clinical signs of S. suis infection, including rough hair coat, weight loss, depression, shivering, and suppuration of the eyes. There were no survivors at 12 hours post-infection (Figure 5). However, mice in the $\Delta$ virB1-89K mutant group were all alive at 12 hours post-infection and had a survival rate of $70 \%$ at the experimental end point of 7 days. When mice were challenged with the complemented strain, CAvirB1-89K, data similar to those obtained with the wild-type strain were observed. In the THY control group, all mice survived without any disease symptoms during the entire experiment. These results strongly indicated that VirB1-89K is involved in the pathogenesis of Chinese epidemic S. suis 2 strains.

\section{Discussion}

T4SSs are versatile devices that are found in many bacterial pathogens and secrete a wide variety of substrates, from single protein to protein-protein and protein-DNA complexes. They are generally composed of a dozen components that are organized into ATP-powered protein complexes spanning the entire cell envelope. In this macromolecular secretion apparatus, the VirB1 component can lysis cell wall peptidoglycan of the bacteria to facilitate the assembly of T4SS [23]. Many VirB1 components in gramnegative bacteria are lytic transglycosylases that can cleave the $\beta-1,4$ glycosidic bond between $\mathrm{N}$-acetylglucosamine (GlcNAc) and N-acetylmuramic acid (MurNAc), with the concomitant formation of a $\beta$-1,6-anhydromuramoyl product [24-27]. In some cases, the VirB1 orthologs can be $\mathrm{N}$-acetylmuramoyl-L-alanine amidases that cleave the link between $\mathrm{N}$-acetylmuramoyl residues and $\mathrm{L}$-amino acid residues in certain cell wall glycopeptides [28]. In this study, sequence alignment and phylogenetic analysis showed that the VirB1-89K protein may be an N-acetylmuramoyl-Lalanine amidase.

To explore the potential role of VirB1-89K in S. suis, we engineered the CHAP domain of VirB1-89K for heterologous expression for three reasons: i) the total length of VirB1-89K is as long as 933 amino acids located within a transmembrane region, suggesting it may be difficult to express and purify the full-length VirB1-89K protein; ii) the main function of a certain protein depends on its
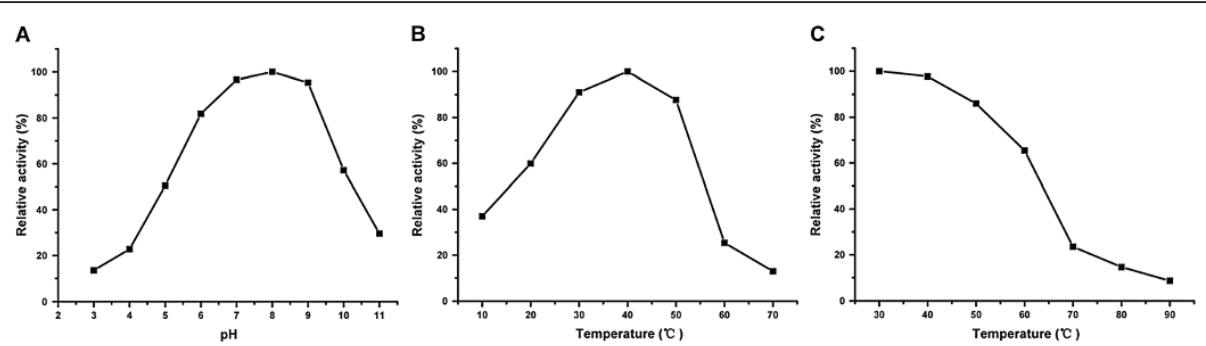

Figure 4 Dynamic changes in lytic activity of VirB1-89KCHAP at different pH values or temperatures. (A) The effect of pH on enzyme activity of VirB1-89KCHAP. (B) The effect of temperature on enzyme activity of VirB1-89KCHAP. (C) Thermostability of the VirB1-89KCHAP protein. Results shown are representative of three independent experiments. 


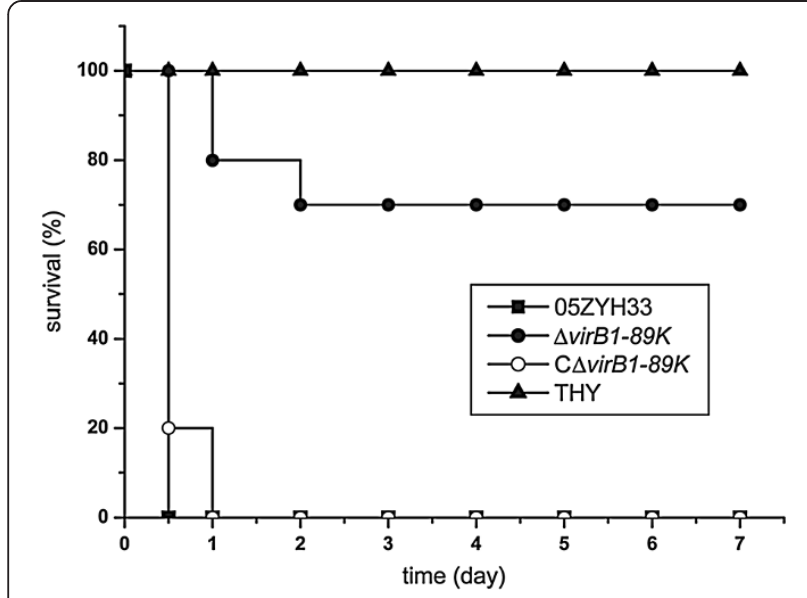

Figure 5 Survival curves of mice infected with S. suis 05ZYH33, the $\Delta$ virB1-89K mutant, the complemented strain C $\Delta$ virB1-89K, and the THY medium. Mice (10 per group) were inoculated intraperitoneally with $10^{8} \mathrm{CFU}$ bacteria. Results shown are representative of three independent experiments.

active domain; iii) recent studies showed that the activity of the functional domain may be higher than the full-length protein [29]. By using S. suis peptidoglycan as the substrate for zymogram analysis, we visually detected the muramidase activity of the purified VirB1-89KCHAP protein. In addition, the bacteriostatic activity of VirB1-89KCHAP was also observed with slip diffusion method. These data confirmed the peptidoglycan hydrolase activity of VirB189KCHAP, indicating the VirB1-89K component may play a crucial role in piercing the peptidoglycan layer in the cell wall of S. suis 2 during the assembly of the T4SS transenvelope transporter complex.

Recently, we reported that the T4SS encoded within the 89K PAI not only contributes to the development of STSS [13], but also mediates the conjugal transfer of $89 \mathrm{~K}$ itself [12]. The transfer frequency of $89 \mathrm{~K}$ was reduced approximately 6 -fold in a virB1-89K deletion mutant ( $\triangle$ virB1-89K) [12]. In this study, we found that the virulence of the $\Delta v i r B 1-89 K$ mutant was reduced to $30 \%$ compared to the wild-type level. A similar phenomenon had been reported that the virB1 defection in A. tumefaciens can cause a marked reduction of virulence to $1 \%-10 \%$ of the wild-type level $[25,30]$. These results indicated that the VirB1 orthologs are important for a functional T4SS, their absence would disturb the proper assembly of the transenvelope apparatus, thus leading to unsuccessful release of the T4SS substrates.

Recent studies suggested that Cagy, the Helicobacter pylori homologue of VirB1, is essential for the CagA effector translocation [31]. However, little is known about the effectors delivered by the S. suis T4SS that are responsible for STSS. Work currently underway in our laboratory seeks to determine these pathogenic effectors.
Furthermore, our future research will focus on the difference in crystal structure between the VirB1 component in gram-negative A. tumefaciens and its counterpart in grampositive S. suis, thus facilitating our understanding of the assembly of the T4SS apparatus in gram-positive bacteria.

\section{Conclusions}

In summary, we characterized a functional peptidoglycan hydrolase from T4SS in the 89K PAI of Chinese epidemic S. suis 2 . In the operon coding for the $89 \mathrm{~K}$ T4SS, the virB1-89K gene product is the only one that shows similarity to the Agrobacterium VirB1 component and contains a conserved CHAP domain. In this work, the purified CHAP domain of VirB1-89K exhibited evident peptidoglycan-degrading and bacteriostatic activity in vitro. Inactivation of virB1-89K reduces significantly the virulence of $S$. suis in a mouse infection model. The experimental results indicated that VirB1-89K facilitates the assembly of 89K T4SS apparatus by breaking apart the peptidoglycan cell wall, thus contributing to the horizontal transfer of $89 \mathrm{~K}$ and the pathogenesis of T4SS in S. suis infection.

\section{Methods}

Bacterial strains, plasmids, and growth conditions

The bacterial strains and plasmids used in this study are listed in Table 1. S. suis strains were grown in Todd-Hewitt broth (THB) supplemented with $2 \%$ yeast extract (THY). E. coil and M. lysodeikticus strains were cultured in LuriaBertani (LB) medium at $37^{\circ} \mathrm{C}$. Solid medium was prepared by the addition of $1.5 \%$ agar. When necessary, antibiotics were added at the following concentrations: spectinomycin, $100 \mu \mathrm{g} / \mathrm{ml}$ for both S. suis and E. coli; chloramphenicol, $5 \mu \mathrm{g} / \mathrm{ml}$ for S. suis and $10 \mu \mathrm{g} / \mathrm{ml}$ for E. coli; ampicillin, $100 \mu \mathrm{g} / \mathrm{ml}$ for E. coli.

\section{Bioinformatics analysis and functional prediction of VirB1-89K}

Sequences were analyzed by using the DNAStar software package. Sequence alignment was performed by using BLAST at NCBI (http://www.ncbi.nlm.nih.gov/blast/). The conserved domain of VirB1-89K was analyzed using the Pfam online server (http://pfam.sanger.ac.uk/). The presence and location of signal peptide was predicted by SignalP 3.0 server (http://www.cbs.dtu.dk/services/SignalP/). The tertiary structure of the conserved domain was determined using SWISS-MODEL web server (http:// swissmodel.expasy.org/) and the PyMOL viewer software. Phylogenetic analysis of VirB1-89K was conducted using the MEGA version 5.1 program.

\section{Cloning, expression, and purification of VirB1-89KCHAP}

A 411 bp fragment encoding the CHAP domain of VirB1$89 \mathrm{~K}$ was amplified from $S$. suis $05 \mathrm{ZYH} 33$ genomic DNA with the forward (5'-GAGACATATGGATTTTTTTGA 


\begin{tabular}{|c|c|c|}
\hline Strains/plasmids & Relevant characteristics $^{*}$ & Source/reference \\
\hline \multicolumn{3}{|l|}{ Strains } \\
\hline \multicolumn{3}{|l|}{ S. suis } \\
\hline 05ZYH33 & A highly virulent strain isolated from a dead patient with STSS & Lab collection \\
\hline$\Delta$ virB1-89K & An isogenic virB1-89K mutant of strain 05ZYH33; Spc ${ }^{r}$ & [12] \\
\hline$C \Delta$ virB1-89K & Complemented strain of $\Delta$ virB $1-89 K_{;} \mathrm{Spc}^{r} ; \mathrm{Cm}^{r}$ & [12] \\
\hline \multicolumn{3}{|l|}{ M. lysodeikticus } \\
\hline ATCC4698 & Suitable for substrate for the assay of lysozyme & Sigma-Aldrich \\
\hline \multicolumn{3}{|l|}{ E. coli } \\
\hline $\mathrm{DH} 5 \mathrm{a}$ & Cloning host for maintaining the recombinant plasmids & Lab collection \\
\hline BL21(DE3) & Expression host for exogenous protein production & Lab collection \\
\hline \multicolumn{3}{|l|}{ Plasmids } \\
\hline pMD19-T & Cloning vector; $\mathrm{Amp}^{\mathrm{r}}$ & TaKaRa \\
\hline pET-21a(+) & His-tag fusion expression vector; $A m p^{r}$ & Novagen \\
\hline pET21a-CHAP & $\begin{array}{l}\text { A recombinant vector with the background of pET-21a(+), } \\
\text { designed for expression of the CHAP domain of VirB1-89K; Amp }\end{array}$ & This work \\
\hline
\end{tabular}

${ }^{*} \mathrm{Amp}{ }^{r}$, ampicillin resistant; $\mathrm{Cm}^{\mathrm{r}}$, chloramphenicol resistant; $\mathrm{Spc}^{\mathrm{r}}$, spectinomycin resistant.

AAACTCTAT-3') and the reverse (5'-GAGACTCGAG TTTCGTCGTATAAGCAAAAC-3') primers carrying the Nde I and Xho I restriction sites, respectively. The resulting PCR products were cloned into the appropriate sites of the pET-21a $(+)$ plasmid, creating the recombinant expression vector pET21a-CHAP. A single colony of E. coli BL21(DE3) containing pET21a-CHAP was inoculated in LB medium and grew overnight, then diluted 1:100 into $2 \mathrm{~L}$ of $\mathrm{LB}$ medium and was grown at $37^{\circ} \mathrm{C}$ to an $\mathrm{OD}_{600}$ of 0.6. Induce cells with IPTG to a final concentration of $1 \mathrm{mM}$ and grow the cultures at $16^{\circ} \mathrm{C}$ for an additional 10 hours. Cells were harvested by centrifugation at 6,000 rpm for $15 \mathrm{~min}$, and the pellet was resuspended in $100 \mathrm{ml}$ binding buffer $\left(20 \mathrm{mM} \mathrm{Na} \mathrm{PO}_{4}, 0.5 \mathrm{M} \mathrm{NaCl}\right.$, $\mathrm{pH}$ 8.0), and then ultrasonic treatment was performed on ice. The supernatant was collected by centrifugation, and the elution buffer $\left(20 \mathrm{mM} \mathrm{Na}_{3} \mathrm{PO}_{4}, 0.5 \mathrm{M} \mathrm{NaCl}, 0.5 \mathrm{M}\right.$ imidazole, $\mathrm{pH}$ 8.0) in accordance with 1:20 were added. The protein of interest (VirB1-89KCHAP) was purified on His GraviTrap column prepacked with Ni Sepharose 6 Fast Flow, then washed with binding buffer until the absorbance reaches the baseline. The target protein was eluted with elution buffer using a linear gradient. The elution was checked by SDS-PAGE (12\%) and fractions containing the interest protein were further purified by gel filtration chromatography using Superdex-75 column. Peak elution fractions were analyzed by gel electrophoresis and those containing pure protein were pooled and concentrated in an Amicon apparatus (Millipore) with a $10-\mathrm{kDa}$ molecular weight cutoff membrane, then stored in $0.1-\mathrm{ml}$ aliquots at $-80^{\circ} \mathrm{C}$. The protein concentration was determined by using the Pierce BCA protein assay kit.

\section{Determination of the lytic activity of VirB1-89KCHAP}

To determine the peptidoglycan-degrading activity of VirB1-89KCHAP, zymogram analysis was performed as described previously [32,33]. Peptidoglycan isolated and purified from $S$. suis 2 was added into $12 \%$ polyacrylamide gels to a final concentration of $100 \mathrm{mg} / \mathrm{ml}[24,34]$. After electrophoresis, the gels were incubated at $37^{\circ} \mathrm{C}$ in renaturation buffer ( $20 \mathrm{mM}$ sodium phosphate buffer, $0.1 \%$ Trition $\mathrm{X}-100,10 \mathrm{mM} \mathrm{MgCl}$, $\mathrm{pH}$ 8.0) for $16 \mathrm{~h}$, and then stained with $1 \%$ methylene blue containing $0.1 \% \mathrm{KOH}$. The deionized water was used for depolarization.

The bacteriostatic activity of VirB1-89KCHAP was determined with slip-agar diffusion method [35]. A small piece of filter paper loaded with purified VirB1-89KCHAP was placed on a $1.5 \%$ agar plate inoculated with S. suis 2 cells, and then bacteriostatic rings of protein-sensitive slips were generally observed after incubation and the diameters of bacteriostatic rings were measured with a vernier caliper. Hen egg white lysozyme and BSA were used as positive and negative controls, respectively.

\section{The effect of $\mathrm{pH}$ and temperature on the enzymatic activity of VirB1-89KCHAP}

The effect of $\mathrm{pH}$ and temperature on the enzymatic activity of VirB1-89KCHAP was determined as previously described with minor modifications [31]. Purified VirB1-89KCHAP protein was added to $200 \mu$ l the dried cells of $M$. lysodeikticus as substrate. To determine the optimal $\mathrm{pH}$ value, the enzyme activity was monitored at $37^{\circ} \mathrm{C}$ with different $\mathrm{pH}$ values ranging from 3.0 to 11.0. The optimum temperature of the enzyme was tested at the temperature ranging from $20^{\circ} \mathrm{C}$ to $70^{\circ} \mathrm{C}$ at 
the optimum $\mathrm{pH}$ value. For the thermal stability estimation, the enzyme was pre-incubated at temperatures between $30^{\circ} \mathrm{C}$ and $90^{\circ} \mathrm{C}$ for $30 \mathrm{~min}$, and the remaining activity was determined under the optimum reaction conditions.

\section{In vivo virulence studies}

To determine whether the virB1-89K gene is necessary for the virulence of the highly pathogenic S. suis 2, experimental infection was performed as previously described [36]. Randomized groups of $10 \mathrm{BALB} / \mathrm{c}$ mice (4-week-old, female) were challenged intraperitoneally with the wild-type, the isogenic knockout mutant of virB1-89K ( $\triangle$ virB1-89K), and the complementary strain CAvirB1-89K, at a dose of $10^{8} \mathrm{CFU}(0.1 \mathrm{ml}$ of each strain) respectively. In parallel, another group of mice was injected with the same volume of THY medium as a negative control. Mice were monitored for clinical signs and survival time for 7 days. All the experiments were approved by the Laboratory Animal Welfare and Ethics Committee of the Third Mililary Medical University.

\section{Statistical analysis}

Where appropriate, the data were analyzed using Student's $t$-test, and a value of $P<0.05$ was considered significant.

\section{Competing interests}

The authors declare that they have no competing interests.

\section{Authors' contributions}

$\mathrm{ML}$ and $\mathrm{FH}$ conceived of the study, and $\mathrm{JT}$ participated in its design and coordination. QZ, YZ, TC, SY, JW, SL, and YT participated in the experiments. $\mathrm{XY}$ and $\mathrm{BZ}$ performed the sequence analysis. $\mathrm{QZ}$ and $\mathrm{ML}$ drafted the manuscript. All authors read and approved the final manuscript.

\section{Acknowledgements}

This work was supported by National Natural Science Foundation of China (No. 31370169 \& 81301398), Program for young medical and scientific scholars of PLA (No. 13QNP106), and Zhejiang Provincial Natural Science Foundation of China (No. LQ13H190002).

\section{Author details}

${ }^{1}$ Department of Microbiology, Third Military Medical University, Chongqing 400038, China. ${ }^{2}$ Department of Scientific Research, Daping Hospital, Research Institute of Surgery, Third Military Medical University, Chongqing 400042, China. ${ }^{3}$ PLA Research Institute of Clinical Laboratory Medicine, Nanjing general hospital of Nanjing Military command, Nanjing 210002, China. ${ }^{4}$ State Key Laboratory for Diagnosis and Treatment of Infectious Disease, The First Affiliated Hospital, College of Medicine, Zhejiang University, Hangzhou 310003, China

Received: 20 December 2013 Accepted: 20 March 2014

Published: 22 March 2014

\section{References}

1. Gottschalk M, Xu J, Calzas C, Segura M: Streptococcus suis: a new emerging or an old neglected zoonotic pathogen? Future Microbiol 2010, 5(3):371-391.

2. Segura M: Streptococcus suis: an emerging human threat. J Infect Dis 2009, 199(1):4-6.

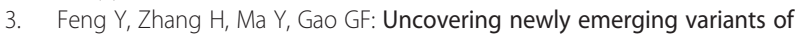
Streptococcus suis, an important zoonotic agent. Trends Microbiol 2010, 18(3):124-131.

4. Huang YT, Teng LJ, Ho SW, Hsueh PR: Streptococcus suis infection. J Microbiol Immunol Infect 2005, 38(5):306-313.
5. Sriskandan S, Slater JD: Invasive disease and toxic shock due to zoonotic Streptococcus suis: an emerging infection in the East? PLOS Med 2006, 3(5):e187.

6. Gottschalk M, Segura M, Xu J: Streptococcus suis infections in humans: the Chinese experience and the situation in North America. Anim Health Res Rev 2007, 8(1):29-45.

7. Tang J, Wang C, Feng $Y$, Yang W, Song H, Chen Z, Yu H, Pan X, Zhou X, Wang $H$, Wu B, Wang $H$, Zhao H, Lin Y, Yue J, Wu Z, He X, Gao F, Khan AH, Wang J, Zhao G, Wang Y, Wang X, Chen Z, Gao GF: Streptococcal toxic shock syndrome caused by Streptococcus suis serotype 2. PLoS Med 2006, 3(5):e151.

8. Yu $H$, Jing $H$, Chen Z, Zheng $H$, Zhu X, Wang $H$, Wang $S$, Liu L, Zu R, Luo L, Xiang N, Liu H, Liu X, Shu Y, Lee SS, Chuang SK, Wang Y, Xu J, Yang W, Streptococcus suis study groups: Human Streptococcus suis outbreak, Sichuan, China. Emerg Infect Dis 2006, 12(6):914-920.

9. Breiman RFDJ, Facklam RR, Gray BM, Hoge CW, Kaplan EL, Mortimer EA, Schlievert PM, Schwartz B, Stevens DL, Todd JK: Defining the group A streptococcal toxic shock syndrome: rationale and consensus definition: the working group on severe streptococcal infections. Jama 1993, 269(3):390-391.

10. Chen C, Tang J, Dong W, Wang C, Feng Y, Wang J, Zheng F, Pan X, Liu D, Li M, Song $Y$, Zhu $X$, Sun $H$, Feng $T$, Guo Z, Ju A, Ge J, Dong $Y$, Sun W, Jiang $Y$, Wang J, Yan J, Yang $H$, Wang $X$, Gao GF, Yang $R$, Wang J, Yu J: A glimpse of streptococcal toxic shock syndrome from comparative genomics of $S$. suis 2 Chinese isolates. PLOS ONE 2007, 2:e315.

11. Li M, Wang C, Feng Y, Pan X, Cheng G, Wang J, Ge J, Zheng F, Cao M, Dong Y, Liu D, Wang J, Lin Y, Du H, Gao GF, Wang X, Hu F, Tang J: SalK/ SalR, a two-component signal transduction system, is essential for full virulence of highly invasive Streptococcus suis serotype 2. PLoS One 2008, 3(5):e2080.

12. Li M, Shen X, Yan J, Han H, Zheng B, Liu D, Cheng H, Zhao Y, Rao X, Wang C, Tang J, Hu F, Gao GF: Gl-type T4SS-mediated horizontal transfer of the 89K pathogenicity island in epidemic Streptococcus suis serotype 2. Mol Microbiol 2011, 79(6):1670-1683.

13. Zhao Y, Liu G, Li S, Wang M, Song J, Wang J, Tang J, Li M, Hu F: Role of a type IV-like secretion system of Streptococcus suis 2 in the development of streptococcal toxic shock syndrome. J Infect Dis 2011, 204(2):274-281.

14. Alvarez-Martinez CE, Christie PJ: Biological diversity of prokaryotic type IV secretion systems. Microbiol Mol Biol Rev 2009, 73(4):775-808.

15. Abajy MY, Kopec J, Schiwon K, Burzynski M, Doring M, Bohn C, Grohmann E: A type IV-secretion-like system is required for conjugative DNA transport of broad-host-range plasmid pIP501 in gram-positive bacteria. J Bacteriol 2007, 189(6):2487-2496.

16. Grohmann E, Muth G, Espinosa M: Conjugative plasmid transfer in gram-positive bacteria. Microbiol Mol Biol Rev 2003, 67(2):277-301. table of contents.

17. Zahrl D, Wagner M, Bischof K, Bayer M, Zavecz B, Beranek A, Ruckenstuhl C, Zarfel GE, Koraimann G: Peptidoglycan degradation by specialized lytic transglycosylases associated with type III and type IV secretion systems. Microbiology 2005, 151(Pt 11):3455-3467.

18. Fronzes $\mathrm{R}$, Christie PJ, Waksman G: The structural biology of type IV secretion systems. Nat Rev Microbiol 2009, 7(10):703-714

19. Bateman A, Rawlings ND: The CHAP domain: a large family of amidases including GSP amidase and peptidoglycan hydrolases. Trends Biochem Sci 2003, 28(5):234-237.

20. Rigden DJ, Jedrzejas MJ, Galperin MY: Amidase domains from bacterial and phage autolysins define a family of gamma-D, L-glutamate-specific amidohydrolases. Trends Biochem Sci 2003, 28(5):230-234.

21. Donovan DM, Foster-Frey J, Dong S, Rousseau GM, Moineau S, Pritchard DG: The cell lysis activity of the Streptococcus agalactiae bacteriophage B30 endolysin relies on the cysteine, histidine-dependent amidohydrolase/ peptidase domain. App/ Environ Microbiol 2006, 72(7):5108-5112.

22. Frankel MB, Hendrickx AP, Missiakas DM, Schneewind O: LytN, a murein hydrolase in the cross-wall compartment of Staphylococcus aureus, is involved in proper bacterial growth and envelope assembly. J Biol Chem 2011, 286(37):32593-32605.

23. Baron C, Llosa M, Zhou S, Zambryski PC: VirB1, a component of the T-complex transfer machinery of Agrobacterium tumefaciens, is processed to a C-terminal secreted product, VirB1. J Bacterio/ 1997, 179(4):1203-1210.

24. Blackburn NT, Clarke AJ: Assay for lytic transglycosylases: a family of peptidoglycan lyases. Anal Biochem 2000, 284(2):388-393. 
25. Mushegian AR, Fullner K, Koonin EV, Nester EW: A family of lysozyme-like virulence factors in bacterial pathogens of plants and animals. Proc Natl Acad Sci U S A 1996, 93(14):7321-7326.

26. Holtje JV, Mirelman D, Sharon N, Schwarz U: Novel type of murein transglycosylase in Escherichia coli. J Bacteriol 1975, 124(3):1067-1076.

27. Koraimann G: Lytic transglycosylases in macromolecular transport systems of Gram-negative bacteria. Cell Mol Life Sci 2003, 60(11):2371-2388.

28. Arends K, Celik EK, Probst I, Goessweiner-Mohr N, Fercher C, Grumet L, Soellue C, Abajy MY, Sakinc T, Broszat M, Schiwon K, Koraimann G, Keller W, Grohmann E: TraG encoded by the pIP501 type IV secretion system is a two-domain peptidoglycan-degrading enzyme essential for conjugative transfer. J Bacteriol 2013, 195(19):4436-4444.

29. Mao J, Schmelcher M, Harty WJ, Foster-Frey J, Donovan DM: Chimeric Ply187 endolysin kills Staphylococcus aureus more effectively than the parental enzyme. FEMS Microbiol Lett 2013, 342(1):30-36.

30. Berger BR, Christie PJ: Genetic complementation analysis of the Agrobacterium tumefaciens virB operon: virB2 through virB11 are essential virulence genes. J Bacteriol 1994, 176(12):3646-3660.

31. Zhong Q, Shao S, Mu R, Wang H, Huang S, Han J, Huang H, Tian S: Characterization of peptidoglycan hydrolase in Cag pathogenicity island of Helicobacter pylori. Mol Biol Rep 2011, 38(1):503-509.

32. Leber TM, Balkwill FR: Zymography: a single-step staining method for quantitation of proteolytic activity on substrate gels. Anal Biochem 1997, 249(1):24-28.

33. Strating $\mathrm{H}$, Clarke AJ: Differentiation of bacterial autolysins by zymogram analysis. Anal Biochem 2001, 291(1):149-154.

34. Clarke AJ: Extent of peptidoglycan $\mathrm{O}$ acetylation in the tribe Proteeae. J Bacteriol 1993, 175(14):4550-4553.

35. Yuan Y, Peng Q, Gao M: Characteristics of a broad lytic spectrum endolysin from phage BtCS33 of Bacillus thuringiensis. BMC Microbiol 2012, 12:297.

36. Wang H, Shen X, Zhao Y, Wang M, Zhong Q, Chen T, Hu F, Li M: Identification and proteome analysis of the two-component VirR/VirS system in epidemic Streptococcus suis serotype 2. FEMS Microbiol Lett 2012, 333(2):160-168.

doi:10.1186/1471-2180-14-73

Cite this article as: Zhong et al:: A functional peptidoglycan hydrolase characterized from T4SS in $89 \mathrm{~K}$ pathogenicity island of epidemic Streptococcus suis serotype 2. BMC Microbiology 2014 14:73.

\section{Submit your next manuscript to BioMed Central and take full advantage of:}

- Convenient online submission

- Thorough peer review

- No space constraints or color figure charges

- Immediate publication on acceptance

- Inclusion in PubMed, CAS, Scopus and Google Scholar

- Research which is freely available for redistribution

Submit your manuscript at www.biomedcentral.com/submit 